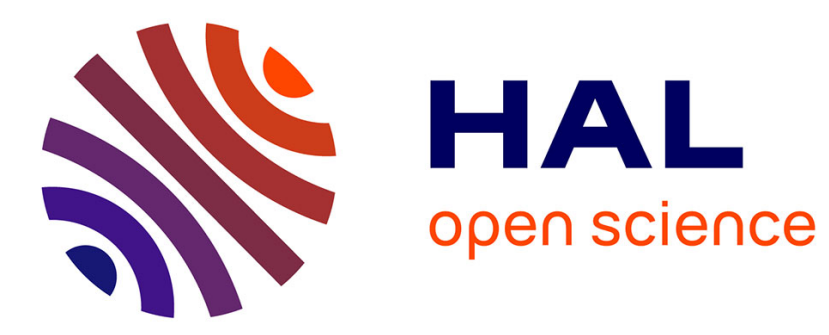

\title{
Reproduction of self-rotation duration
}

I. Israël, I. Siegler, S. Rivaud-Péchoux, B. Gaymard, P. Leboucher, M.

Ehrette, A. Berthoz, C. Pierrot-Deseilligny, T. Flash

\section{To cite this version:}

I. Israël, I. Siegler, S. Rivaud-Péchoux, B. Gaymard, P. Leboucher, et al.. Reproduction of selfrotation duration. Neuroscience Letters, 2006, 402 (3), pp.244-248. 10.1016/j.neulet.2006.04.005 . hal-02395714

\section{HAL Id: hal-02395714 https://hal.science/hal-02395714}

Submitted on 5 Dec 2019

HAL is a multi-disciplinary open access archive for the deposit and dissemination of scientific research documents, whether they are published or not. The documents may come from teaching and research institutions in France or abroad, or from public or private research centers.
L'archive ouverte pluridisciplinaire HAL, est destinée au dépôt et à la diffusion de documents scientifiques de niveau recherche, publiés ou non, émanant des établissements d'enseignement et de recherche français ou étrangers, des laboratoires publics ou privés. 


\title{
Reproduction of self-rotation duration
}

\author{
I. Israël ${ }^{\mathrm{a}, *}$, I. Siegler ${ }^{\mathrm{a}}$, S. Rivaud-Péchoux ${ }^{\mathrm{b}}$, B. Gaymard ${ }^{\mathrm{b}}$, P. Leboucher ${ }^{\mathrm{a}}$, \\ M. Ehrette ${ }^{\mathrm{a}}$, A. Berthoz ${ }^{\mathrm{a}}$, C. Pierrot-Deseilligny ${ }^{\mathrm{b}}$, T. Flash $^{\mathrm{c}}$ \\ a LPPA, CNRS-Collège de France, Paris, France \\ ${ }^{\mathrm{b}}$ INSERM-U679, Hôpital de la Salpêtrière, Paris, France \\ ${ }^{\mathrm{c}}$ Weitzmann Institute of Science, Rehovot, Israël
}

Received 27 October 2005; received in revised form 27 March 2006; accepted 8 April 2006

\begin{abstract}
The vestibular system detects the velocity of the head even in complete darkness, and thus contributes to spatial orientation. However, during vestibular estimation of linear passive self-motion distance in darkness, healthy human subjects mainly rely on time, and they replicate also stimulus duration when required to reproduce previous self-rotation. We then made the hypothesis that the perception of vestibular-sensed motion duration is embedded within encoding of motion kinetics. The ability to estimate time during passive self-motion in darkness was examined with a self-rotation reproduction paradigm. Subjects were required to replicate through self-driven transport the plateau velocity $(30,60$ and $90 \%$ s) and duration ( 2,3 and $4 \mathrm{~s}$ ) of the previously imposed whole-body rotation (trapezoid velocity profile) in complete darkness; the rotating chair position was recorded $(500 \mathrm{~Hz})$ during the whole trials. The results showed that the peak velocity, but not duration, of the plateau phase of the imposed rotation was accurately reproduced. Suspecting that the velocity instruction had impaired the duration reproduction, we added a control experiment requiring subjects to reproduce two successive identical rotations separated by a momentary motion interruption (MMI). The MMI was of identical duration to the previous plateau phase. MMI duration was fidelitously reproduced whereas that of the plateau phase was hypometric (i.e. lesser reproduced duration than plateau) suggesting that subjective time is shorter during vestibular stimulation. Furthermore, the accurate reproduction of the whole motion duration, that was not required, indicates an automatic process and confirms that vestibular duration perception is embedded within motion kinetics.
\end{abstract}

(C) 2006 Published by Elsevier Ireland Ltd.

Keywords: Vestibular system; Time estimate; Self-motion; Duration reproduction

The contribution of the vestibular system to orientation and localization of the body in space has long been suggested, and recent studies have shown that indeed, for passive angular whole-body motion in darkness [3,12,13,16,21,22], the brain can retrieve the traveled path amplitude from vestibular information (together with somatosensory information). These estimates of angular self-motion are probably computed first by time integrations (from the vestibular signal of acceleration to position) and then through the more topologic (spatial and temporal) "path integration" $[17,18]$.

However, in our experiments on distance estimation of linear transport [2,11], subjects used time (they mentally counted) to complement vestibular information in order to evaluate their self-motion magnitude. Furthermore, when required to repro-

\footnotetext{
* Correspondence to: LDC-EPHE, 41 rue Gay-Lussac, 75005 Paris, France. Tel.: +33144107816; fax: +33143268816.

E-mail address: isi@ccr.jussieu.fr (I. Israël).
}

duce self-rotation amplitude, subjects reproduced also motion duration (and peak velocity) [10,21]. We then made the hypothesis that time was reproduced "automatically", i.e. that the perception of vestibular-sensed motion duration is embedded within the encoding of motion kinetics and cannot be processed independently during motion.

Subjects were required to reproduce the plateau duration of the previously imposed rotation, in complete darkness. In order to examine also the effect of motion velocity on time estimate, different plateau velocities were used, and the subjects had to replicate both the duration and the velocity of the plateau. The aim of the plateau segment (stimulus shorter than $5 \mathrm{~s}$ ) was to provide target time intervals start/end cues through the motion inertial acceleration steps per se, thus without additional sensory input in order to avoid the possible different sensory modality influence [19] on vestibular time estimation.

Sixteen healthy volunteers (eight men and eight women), aged 19-41 years, participated in this study which was accepted 
by the local ethics committee, and all signed an informed consent prior participation.

Subjects were seated on a motorized rotating chair turning about the vertical axis. The instant angular position was recorded $(500 \mathrm{~Hz})$, from which velocity and acceleration were computed off-line. The subjects could be passively moved (stimulus) with the chair driven by a PC micro-computer, or they could drive themselves the chair with a joystick controlling rotational velocity (response). The experiment was performed in complete darkness, and the subjects wore a walkman delivering wide-band noise in order to mask external auditory cues.

The task was to reproduce in the same direction (clockwise) the previously imposed rotation, which had a trapezoid velocity profile (Table 1, Fig. 1A and B): three plateau velocities (PV: 30, 60 and 90), and three plateau durations (PD: 2, 3 and $4 \mathrm{~s}$ ) contingent to three acceleration steps $(40,50$ and $60 \% \mathrm{~s}^{2}$ ) were applied, leading to nine different angles (range $82-496^{\circ}$ ). Those nine trials were repeated four times in random order. Subjects were instructed to reproduce specifically the
Table 1

Stimuli

\begin{tabular}{llllllc}
\hline PV $(\% / s)$ & Ac $\left(\% \mathrm{~s}^{2}\right)$ & TTP $(\mathrm{s})$ & PDur $(\mathrm{s})$ & TAng $\left({ }^{\circ}\right)$ & TDur $(\mathrm{s})$ & PAng $\left({ }^{\circ}\right)$ \\
\hline 30 & 40 & 0.6 & 2 & 82 & 3.3 & 63.5 \\
30 & 50 & 0.5 & 3 & 108.5 & 4.1 & 91.9 \\
30 & 60 & 0.4 & 4 & 136 & 4.9 & 122.6 \\
60 & 40 & 1.2 & 2 & 210 & 4.5 & 127.6 \\
60 & 50 & 1.1 & 3 & 253 & 5.3 & 180.4 \\
60 & 60 & 0.8 & 4 & 301 & 5.7 & 250.4 \\
90 & 40 & 1.9 & 2 & 383 & 6.1 & 196.6 \\
90 & 50 & 1.6 & 3 & 433 & 6.3 & 271.9 \\
90 & 60 & 1.1 & 4 & 497 & 6.4 & 381.7 \\
\hline
\end{tabular}

Description of the stimuli: plateau velocity (PV), acceleration (Ac), timeto-plateau (TTP), plateau duration (PDur), total angle (TAng), total duration (TDur), and plateau angle (PAng).

plateau velocity and duration of the stimulus motion. The maximal velocity of the responses was $100 \%$, and joystick driven chair acceleration was $80 \% \mathrm{~s}^{2}$. Subjects were told that the more they would push the joystick the faster the chair would rotate,
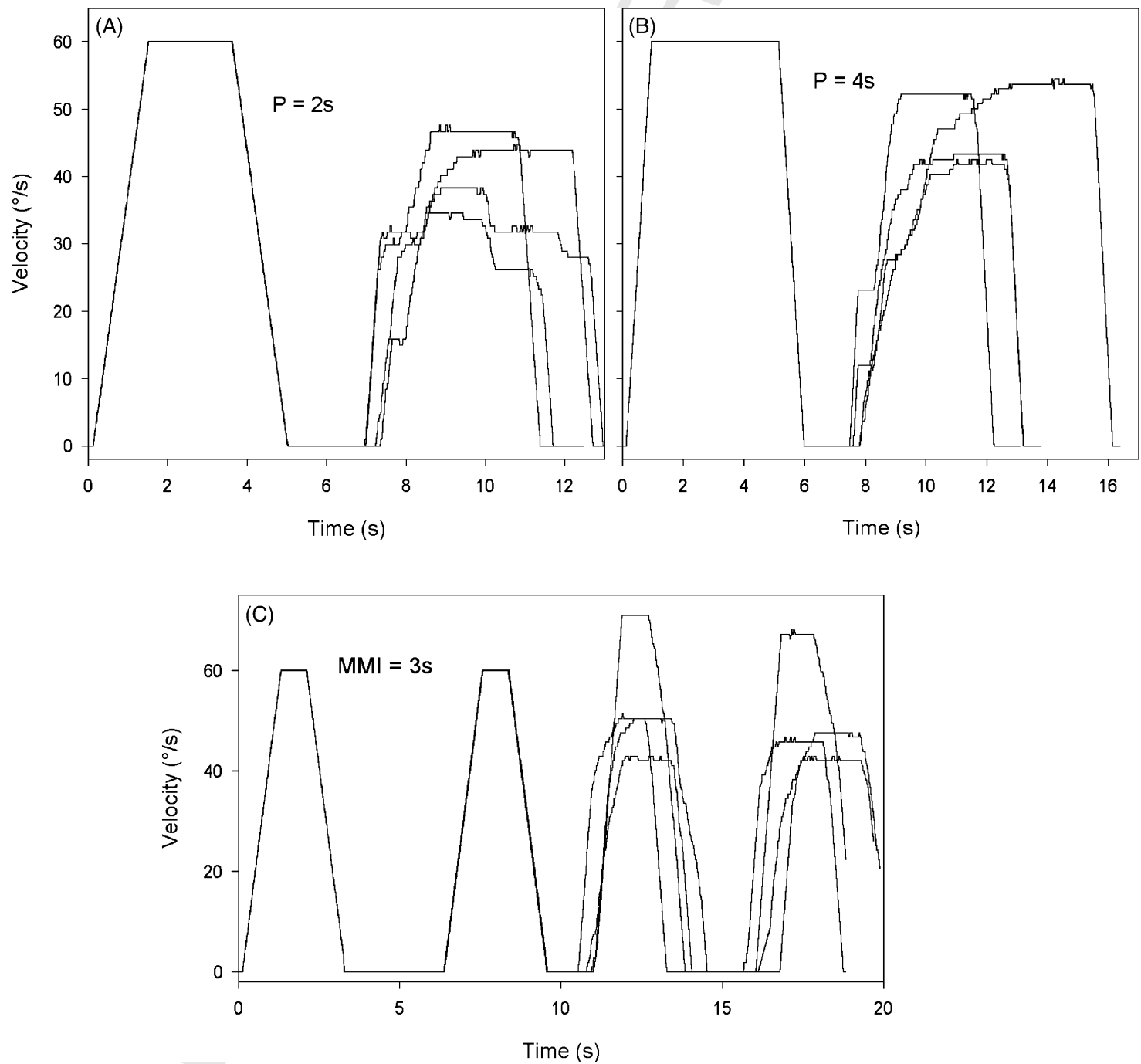

Fig. 1. Sample velocity recordings: (A) plateau duration $=2 \mathrm{~s}$, plateau velocity $=60 \%$. The four repeated trials are shown, with the stimulus on the left and responses on the right; (B) plateau duration $=4 \mathrm{~s}$, plateau velocity $=60 \%$ s. Same subject as in A. It can be seen that the subject did not reach the plateau at once, and undershot plateau velocity; and (C) exemple of MMI, with on the left the two stimulus rotations of $60 \%$ seak velocity separated by a $3 \mathrm{~s}$ Momentary Motion Interruption, and the whole pattern reproduced on the right. This subject overshot stimulus velocity in one trial only. 
Table 2

Results

\begin{tabular}{|c|c|c|c|c|c|c|c|}
\hline $\mathrm{PV}(\% / \mathrm{s})$ & GVel & GPDur & GTDur & PD & GVel & GPDur & GTDur \\
\hline 30 & $1.16 \pm 0.08$ & $0.70 \pm 0.09$ & $1.19 \pm 0.06$ & $2 \mathrm{~s}$ & $0.92 \pm 0.04$ & $0.91 \pm 0.08$ & $1.14 \pm 0.05$ \\
\hline 60 & $0.88 \pm 0.04$ & $0.75 \pm 0.06$ & $1.11 \pm 0.04$ & $3 s$ & $0.97 \pm 0.04$ & $0.73 \pm 0.06$ & $1.06 \pm 0.03$ \\
\hline 90 & $0.86 \pm 0.05$ & $0.88 \pm 0.10$ & $0.99 \pm 0.05$ & $4 s$ & $1.02 \pm 0.04$ & $0.68 \pm 0.07$ & $1.09 \pm 0.05$ \\
\hline
\end{tabular}

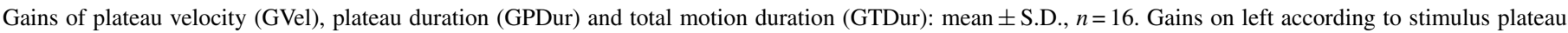
velocity (PV) and on right according to stimulus plateau duration (PD).

and were allowed 5 min practice in darkness before to start the experiment.

As we wanted to determine which variables were more accurately reproduced by the subjects, we used gains (amplitude ratios: response/stimulus), in order to be able to compare the performance also when different variables (of velocity, time, ...) are involved. The four repeated trials were pooled, and repeated measures ANOVAs over stimulus PV (3) $\times$ PD (3) on the different gains were applied.

It can first be seen in Fig. 1A and B that the plateau velocity was not reached in one shot, but subjects apparently hesitated before to adopt a plateau velocity, and then maintained the same velocity until the end of plateau duration. The plateau was defined as the whole area (line) where velocity changes were smaller than $10 \%$ s.

The overall gain of the plateau duration (GPDur) was $0.78 \pm 0.19$ (mean \pm S.D., $n=16$; S.D. of the overall gain is greater than the S.D. at each PD or each PV because the corresponding mean gains were different, Table 2), thus lower than 1 (the ideal gain), and with no difference between men $(0.77 \pm 0.20, n=8)$ and women $(0.79 \pm 0.18)$.

Surprisingly, even for a duration as short as $2 \mathrm{~s}$, the subjects undershot this plateau, although this undershoot was relatively less at $2 \mathrm{~s}$ than at 3 and $4 \mathrm{~s}$ PD (Table 2). While it was always lower than unity, GPDur increased with stimulus $\mathrm{PV}(F[2,30]=11.78, p<0.0002)$. This suggests that subjective time was relatively longer at higher velocities. Furthermore, the slopes of the plateau duration stimulus-response regression lines were significantly different from zero in only eight subjects, and were low $(0.48 \pm 0.15, n=8)$. So globally the subjects did not succeed reproducing the plateau duration.

As the plateau was only a segment of the whole rotation, the reproduction of the total motion duration was also analyzed. The overall total duration gain (GTDur) was $1.09 \pm 0.11$ (thus higher and with smaller S.D. than GPDur). The slopes of the total duration stimulus-response regression lines were different from zero in 14 subjects, and were higher than for the plateau duration $(0.72 \pm 0.13, n=14)$. Duration reproduction is therefore easier for the whole motion than for only a part of it. Unlike GPDur, GTDur was almost always higher than 1. This suggests that the responses were longer than the stimulus, but only before and after the plateau.

This was checked through the time-to-plateau (TTP), corresponding to the motion acceleration phase (Table 1), as well as on the other side of the plateau, i.e. the time-from-plateau (TFP), corresponding to the deceleration phase. The overall TTP gain was $2.34 \pm 0.40$ and TFP gain was $1.35 \pm 0.47$. The high TTP gain shows that the subjects drove softly: it was negatively cor- related $(p=0.0024)$ with a low acceleration gain $(0.62 \pm 0.14)$, and TFP gain was negatively correlated with the deceleration gain $(0.99 \pm 0.26)$. The high gains of TTP and TFP explain why the total duration gain was higher than 1 whereas the plateau duration gain was lower.

The overall gain of plateau velocity (GVel) was $0.97 \pm 0.08$, i.e. very close to 1 . GVel was higher than GPDur ( $t$-test for dependent samples, $t=3.32, p<0.005$ ). It can also be noted that the inter-individual variability (S.D.) of GVel was smaller than for GPDur.

GVel was higher than 1 at the lowest stimulus $\mathrm{PV}(30 \%)$ and lower than 1 at both higher PVs $(F[2,30]=72.30, p<0.0001$; Table 2). But it should be noted that the stimulus angles at $30 \%$ PV were all smaller than a half-turn $\left(180^{\circ}\right)$ and were all larger than $180^{\circ}$ at higher PVs (Table 1): the rotation angle might have influenced the reproduction of its velocity. The slopes of the plateau velocity stimulus-response regression lines were highly significantly different from zero in all subjects $(0.71 \pm 0.12)$.

Furthermore, GVel increased with increasing stimulus PD $(F[2,30]=14.10, p<0.0001$; Table 2$)$. This suggests that longer stimulus plateau duration allowed the subjects reaching a higher plateau velocity.

Because the poor performance in plateau duration reproduction could at least partly be due to the concurrent instruction on plateau velocity, we devised a control test with zero velocity. Five subjects were required to reproduce the duration of the momentary motion interruption (MMI) between two successive identical rotations (Fig. 1C). So as above the subjects replicated the whole stimulus, but it included here two rotations (of 100, 120 , and $140^{\circ}$ ) and the MMI. MMI durations were similar to the previous plateau condition (2-4s). The peak velocity of the two surrounding rotations was $60 \%$ and the acceleration was $50 \% \mathrm{~s}^{2}$, i.e. the characteristics of the previous middle plateau rotation.

The main result of this control experiment was that the MMI duration replication was accurate: the gain was $0.90 \pm 0.18$ $(n=5$; it was $0.95 \pm 0.2,0.88 \pm 0.18$ and $0.87 \pm 0.19$ for MMI of 2, 3 and $4 \mathrm{~s}$, respectively), and the stimulus-response regression slope was $0.79 \pm 0.25$ (different from zero for all subjects). Whereas GPDur $(0.75 \pm 0.06$ at $60 \%)$ and the MMI duration gain were not significantly different, the stimulus-response duration slopes were higher for MMI $(t=2.82, p=0.017)$ than for the eight subjects with a slope different from zero in the plateau experiment. Furthermore, the peak velocity gains of the two surrounding rotations $(0.91 \pm 0.10$ for the first and $0.99 \pm 0.14$ for the second rotation) were not different from the plateau velocity gain. Finally, the duration gains of the surrounding rotations were $0.89 \pm 0.08$ and $0.92 \pm 0.09$ for the first and 
the second rotation, respectively. So as in the plateau experiment, the total duration of motion was accurately reproduced.

The main result of this control experiment is that without motion also responses durations were undershot, but to a much lesser degree than during motion, and the slopes of the stimulus-response regression lines revealed that the MMI duration was indeed replicated, which was not the case during motion.

In this paper we tested the hypothesis that the perception of vestibular-sensed motion duration is embedded within the encoding of motion kinetics. The main result is that the plateau durations of imposed whole-body rotations were not correctly reproduced, while the durations of the total motion, although this was not requested, were accurately reproduced. This supports the idea that perception of vestibular-motion time cannot be encoded via a channel separate of that of motion.

The plateau duration reproductions were always too short, and only half of the subjects exhibited a satisfying plateau duration stimulus-response relationship, while without motion (MMI) all subjects had a significant stimulus-response time relationship. It is known that constant self-motion velocity is not immediately perceived, but that the same time constant applies also to zero-velocity perception after an acceleration step [15].

Furthermore, plateau duration gain (GPDur) tended to increase with stimulus velocity (PV), suggesting that subjective time was longer at higher self-velocity. This supports the results of $[4,20]$ about moving visual stimuli, where faster speeds lengthened reproduced time. But apart of the effect of velocity, the overall small GPDur suggests that the subjective time is shorter during self-motion than without motion (as suggested by the MMI experiment), as it is with timing of visual stimulation relative to auditory stimulation [19].

For the total motion duration, the fact that only two subjects (among 16) did not produce adequate stimulus-response duration regression slopes suggests that total motion duration reproduction is automatic. Furthermore, the mean time gain was far from unity at each motion stage including the plateau where the instruction applied, but was close to 1 and its S.D. was lower for the total duration. So the subjects did not handle the exercise as a sequence of discrete temporal events (although this is what we had expected), but naturally retrieved the overall motion temporal envelope. It should be noted that in this reproduction paradigm, duration criterion is disfavored, since while velocity or angle can be adjusted through corrections in both the up and down directions, time repetition cannot be adjusted in the down direction. This may be one reason why the subjects quasisystematically undershot response duration, both in the plateau and MMI reproductions. But a decay theory of forgetting temporal information in visually-guided movements was suggested [8], which might apply also for non-visually guided movements.

There is an important literature about time estimation and attention, mainly based on dual-tasks [5,7]. However, our task was not a dual one, because the subjects were indeed required to reproduce two concurrent variables of the same motion, and some subjects actually complained about it, but those two variables were not conflicting and, moreover, they were reproduced in sequence. The subjects first attempted to reach the right veloc- ity, and then just had to keep the joystick at the same position during the right duration (Fig. 1A and B). However, we observed the classic interference effect in timing: the concurrent nontemporal task (velocity reproduction) may have contributed to the underestimation of vestibular-sensed motion time reproduction $[6,9]$.

It should also be noted that the stimulus was passive and the response was active (self-driven), while it is known that less attention shortens subjective duration [7,14]. If attention and activity are interdependent, then the lesser attention devoted to the passive stimulus duration (shorter stimulus subjective duration) followed by the higher attention devoted to the active response duration (longer reproduction subjective duration), would combine and magnify response duration undershoot. However, the same argument should also apply to the MMI test, where the responses tended to last longer than with the plateau. So the self-driven plateau response was subjectively shorter than the self-driven immobile response.

Surprisingly, the subjects did not try to reach as quickly as possible the plateau they had to reproduce, since the time-toplateau gain was much higher than unity and the acceleration was low, as if they tried to avoid passing beyond the stimulus velocity. This behavior could explain the second main result, namely that plateau velocity was quite accurately reproduced, according to the gain and the regressions. It can be noted that GVel was higher than unity only at the smallest PV. This could partly be attributed to the range effect, but this smallest PV was also the only stimulus at which the angles were smaller than $180^{\circ}$. This suggests an additional effect of rotation amplitude on velocity reproduction, and brings a new result on the perception of self-rotation velocity $[1,15]$.

It could be argued that the post-rotatory sensations occurring after the stimulus plateau onset distorted its duration estimate, so what was measured was this distortion. But it is well-known that post-rotatory sensations are highly idiosyncratic, and rapidly adapt and decay [21], while no difference was found in the plateau duration reproduction during the experiment (of 36 trials).

Our temporal results showed that the subjects could not reproduce what they had to (plateau duration) and did reproduce what they were not required to (total motion duration). So on one hand the whole motion duration is automatically replicated with motion dynamics, and on the other hand a segment of motion cannot be extracted with all motion properties.

\section{Acknowledgments}

This work was supported by AFIRST (France-Israël), IFR (Hôpital de la Salpêtrière, France), ACI (Ministère de la Recherche, France). We are very grateful to the two anonymous referees whose comments and criticisms greatly helped us in improving the original submission.

\section{References}

[1] W. Becker, R. Jürgens, T. Boss, Vestibular perception of self-rotation in different postures: a comparison between sitting and standing subjects, Exp. Brain Res. 131 (2000) 468-476. 
[2] A. Berthoz, I. Israël, P. Georges-François, R. Grasso, T. Tsuzuku, Spatial memory of body linear displacement: what is being stored? Science 269 (1995) 95-98.

[3] J.J. Bloomberg, G. Melvill Jones, B.N. Segal, Adaptive modification of vestibularly perceived rotation, Exp. Brain Res. 84 (1991) 47-56.

[4] S.W. Brown, Time, change, and motion: the effects of stimulus movement on temporal perception, Percept. Psychophys. 57 (1995) 105-116.

[5] S.W. Brown, Attentional resources in timing: interference effects in concurrent temporal and nontemporal working memory tasks, Percept. Psychophys. 7 (1997) 1118-1140.

[6] S.W. Brown, E.D. Bennett, The role of practice and automaticity in temporal and nontemporal dual-task performance, Psychol. Res. 66 (2002) $80-89$.

[7] L. Casini, F. Macar, Effects of attention manipulation on judgments of duration and of intensity in the visual modality, Mem. Cogn. 25 (1997) 812-818.

[8] D. Elliott, R. Jones, Short-term memory for duration of movement, Percept. Mot. Skills 58 (1984) 331-334.

[9] N.S. Hemmes, B.L. Brown, C.N. Kladopoulos, Time perception with and without a concurrent nontemporal task, Percept. Psychophys. 66 (2004) 328-341.

[10] I. Israël, A. Bronstein, R. Kanayama, M. Faldon, M.A. Gresty, Visual and vestibular factors influencing vestibular "navigation", Exp. Brain Res. 112 (1996) 411-419.

[11] I. Israël, A. Capelli, D. Sablé, C. Laurent, C. Lecoq, J. Bredin, Multifactorial interactions involved in linear self-transport distance estimate: a place for time, Int. J. Psychophysiol. 53 (2004) 21-28.

[12] I. Israël, M. Fetter, E. Koenig, Vestibular perception of passive wholebody rotation about horizontal and vertical axes in humans: goal-directed vestibulo-ocular reflex and vestibular memory-contingent saccades, Exp. Brain Res. 96 (1993) 335-346.

[13] I. Israël, S. Rivaud, B. Gaymard, A. Berthoz, C. Pierrot-Deseilligny, Cortical control of vestibular-guided saccades in man, Brain 118 (1995) 1169-1183.

[14] F. Macar, S. Grondin, L. Casini, Controlled attention sharing influences time estimation, Mem. Cogn. 22 (1994) 673-686.

[15] T. Mergner, A. Rumberger, W. Beckert, Is perceived angular displacement the time integral of perceived angular velocity? Brain Res. Bull. 40 (1996) 467-470.

[16] T. Metcalfe, M.A. Gresty, Self-controlled reorienting movements in response to rotational displacements in normal subjects and patients with labyrinthine disease, Ann. N. Y. Acad. Sci. 656 (1992) 695-698.

[17] M.L. Mittelstaedt, H. Mittelstaedt, Homing by path integration in a mammal, Naturwissenschaften 67 (1980) 566-567.

[18] H. Mittelstaedt, M.L. Mittelstaedt, Homing by path integration, in: F. Papi, H.G. Wallraff (Eds.), Avian Navigation, Springer Verlag, Berlin, Heidelberg, 1982, pp. 290-297.

[19] T.B. Penney, J. Gibbon, W.H. Meck, Differential effects of auditory and visual signals on clock speed and temporal memory, J. Exp. Psychol. Hum. Percept. Perform. 26 (2000) 1770-1787.

[20] J. Predebon, Stimulus motion and retrospective time judgments, Acta Psychol. (Amst.) 109 (2002) 213-225.

[21] I. Siegler, I. Viaud-Delmon, I. Israël, A. Berthoz, Self-motion perception during a sequence of whole-body rotations in darkness, Exp. Brain Res. 134 (2000) 66-73.

[22] L. Yardley, D. Papo, A. Bronstein, M. Gresty, M. Gardner, N. Lavie, L. Luxon, Attentional demands of continuously monitoring orientation using vestibular information, Neuropsychologia 40 (2002) 373-383. 